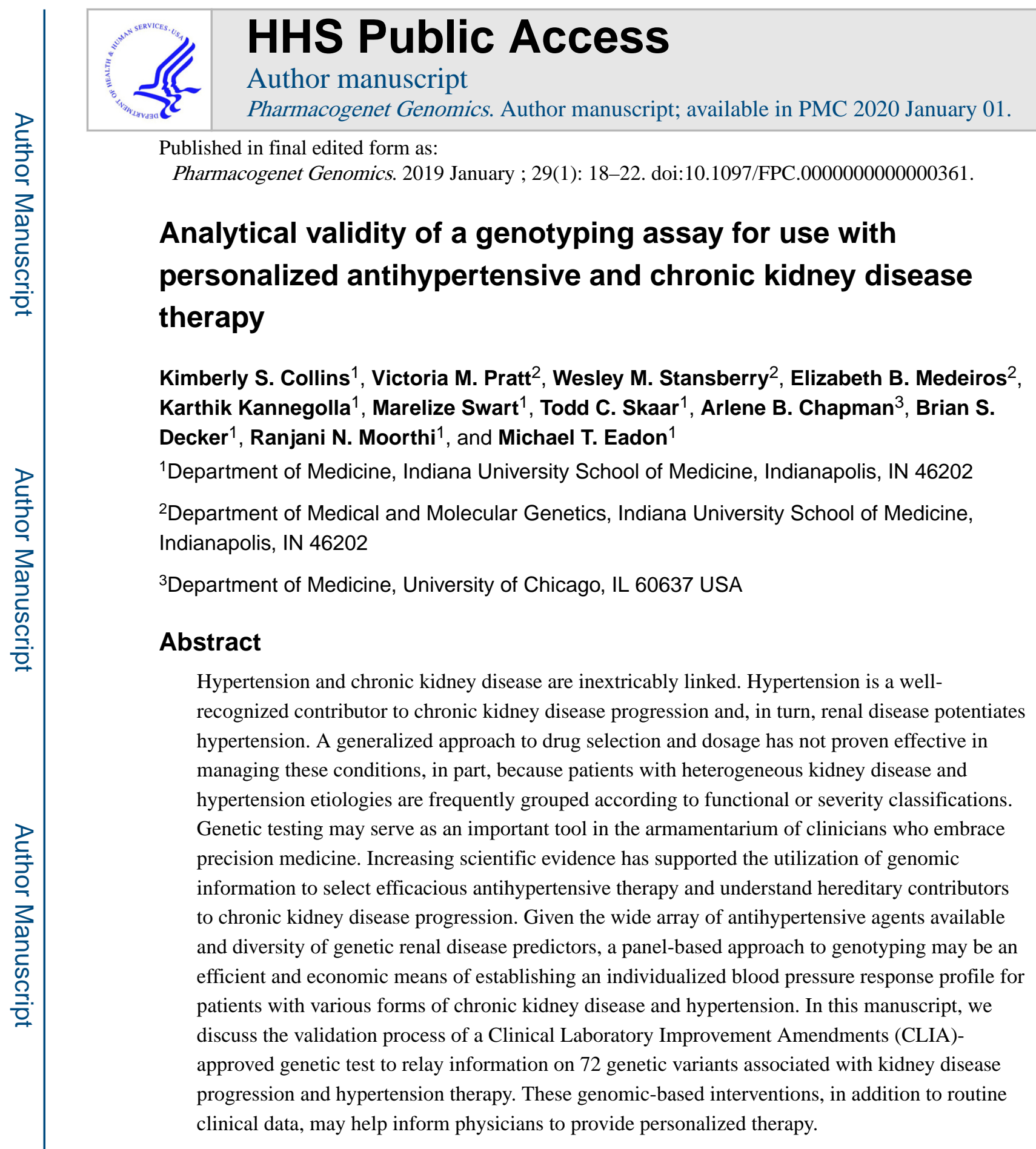

\title{
Introduction
}

Hypertension and chronic kidney disease are intersecting diseases with enormous economic burdens. The design and implementation of genotype-guided care in clinics enables providers to identify drug-gene interactions to inform medication selection, improve treatment efficacy, and reduce adverse events and their associated medical costs. Genetic predictors of chronic kidney disease (CKD) progression may augment standard clinical prediction models, impacting prognosis, medication selection, dialysis initiation, and renal transplant organ allocation. We have designed a genotyping test to detect 72 genetic variants 
in drug metabolizing enzymes and other genes to aid providers in the management of hypertension, chronic kidney disease, and other related conditions.

This communication focuses on the analytical validity of the renal precision medicine assay, including CLIA validation with the assessment of precision and accuracy, analytical sensitivity and specificity, variant reporting strategy, and confirmation of positive reference materials through sequencing. Our goal is to facilitate implementation for future investigators and health systems. A detailed discussion of each variant's clinical validity extends beyond the scope of this manuscript; however, content examples are provided in order to convey context and purpose.

\section{Variant Selection}

Variants in the renal precision medicine assay were selected after balancing their significance, effect size, minor allele frequency, and level of evidence. When applicable, drug-gene phenotypes were utilized from the Pharmacogene Variation Consortium (PharmVar), the Food and Drug Administration (FDA) drug label, Clinical Pharmacogenomics Implementation Consortium (CPIC) and Dutch Pharmacogenetics Working Group (DPWG) [1, 2]. The three groups of variants represented include predictors of 1) antihypertensive drug response, 2) drug response relevant to immunosuppression or cardiovascular disease, and 3) CKD progression. Antihypertensive pharmacogenomic variants were included for metoprolol (CYP2D6), other beta-blockers (GRK4, ADRB1), hydralazine (NAT2), losartan (CYP2C9), amlodipine (F7), thiazide diuretics ( YEATS4, $F G F 5 / S H 2 B 3 / E B F 1)$, diuretics (NEDD4L), angiotensin-converting-enzyme inhibitors (F7), and angiotensin II receptor blockers (NPHS1) (Table 1) [3-11]. The clinical validity of these variants has been previously reviewed and their associated levels of evidence vary $[12,13]$. For example, CYP2D6-guided dosing of metoprolol has been recommended by the DPWG. The FDA drug labels of hydralazine and losartan include NAT2 and CYP2C9 metabolizer status, respectively, as predictors of response. This assay employs genotype as a surrogate to establish metabolizer status for these drugs. Other antihypertensive response predictors included in the assay have lower levels of evidence for implementation. Although they have been replicated in clinical trials, their clinical use is still considered investigational.

A second group of pharmacogenomic variants found in the $C Y P 3 A 4$ and $C Y P 3 A 5, T P M T$, SLCO1B1, CYP2C9, VKORC1, and CYP2C19 genes have been widely studied with genotype-guided dosing recommendations from CPIC and DPWG for drugs such as tacrolimus, azathioprine, simvastatin, warfarin, and clopidogrel (Table 1) [14-18]. The immunosuppressant drug-gene pairs were selected to aid in glomerulonephritis and posttransplant care. The cardiovascular drug-gene pairs were included due to the preponderance of cardiovascular disease in patients with CKD.

A third group of variants have been identified as predictors of CKD progression (Table 2) [19-22]. Genes associated with progression were identified from large genome-wide association studies or prospective clinical cohorts and trials such as the Chronic Renal Insufficiency Cohort and African American Study of Kidney Disease and Hypertension [20]. These genes include APOL1, relevant to individuals with African ancestry, as well as other 
genes identified from African-American, Asian and Caucasian populations, with or without diabetes mellitus. Their clinical validity has been reviewed elsewhere [23].

\section{Variant Reporting}

When feasible, genetic nomenclature and allele reporting was utilized from PharmVar and CPIC. Drug-gene pairs with lower levels of evidence often lack a consensus allelic structure. As a result, the $A D R B 1, F G F 5 / S H 2 B 3 / E B F 1$, and $G R K 4$ genes each include a multi-variant model to predict efficacy that is summarized by copies of amino acids or efficacy alleles. The APOL1 gene is comprised of the $* \mathrm{G} 1(\mathrm{G}$ and $\mathrm{GM})$ and $* \mathrm{G} 2$ variant alleles. The $* \mathrm{G} 1$ allele is described as two missense variants and the ${ }^{*} \mathrm{G} 2$ allele is a 6 base pair deletion that is in high linkage disequilibrium with a single nucleotide polymorphism marker used in this assay. These variants are reported based on quantity of risk alleles. All other variants were classified based on the nucleotides detected. The effects of some variants were only studied or found to be significantly associated with phenotypes in certain populations. As such, some variants are only relevant in a single ethnicity or in individuals without diabetes. This distinction is made in the relevant population column of Table 1 and 2.

\section{Genotyping Assay Validation}

We designed a custom OpenArray in which Taqman allele discrimination was used for genotyping analysis for our selected variants. DNA samples were amplified in singlicate by real-time PCR on the LifeTech QuantStudio 12K Flex (software v1.2.2; Grand Island, NY) using commercially available reagents for the custom designed OpenArray according to the user manual (Life Technologies, Grand Island, NY). DNA samples used for analytical validation were obtained from the Coriell Cell Repository (Camden, NJ) and Icahn School of Medicine at Mount Sinai (New York, NY) [24]. In this paper, we describe the CLIA validation process for the variants provided in Supplemental Table 1, Supplemental Digital Content 1, http://links.lww.com/FPC/B331 . The CYP450s, SLCO1B1, TPMT, and VKORC1 variants have been previously validated as part of the INGENIOUS trial [25].

Since there were no known reference materials for most variants, selected DNA samples were Sanger sequenced with custom designed primers (Integrated DNA Technologies, Coralville, IA) for accuracy studies (Supplemental Table 1, Supplemental Digital Content 1, http://links.lww.com/FPC/B331). PCR amplification was performed on the GeneAmp PCR System 2720 (Applied Biosystems). The targeted gene region was amplified using custom primers and PCR SuperMix (Invitrogen, Carlsbad, CA) at the following PCR amplification conditions: $94^{\circ} \mathrm{C}$ for 2 minutes, 35 cycles at $94^{\circ} \mathrm{C}$ for 15 seconds, $56^{\circ} \mathrm{C}$ for 30 seconds, $72^{\circ} \mathrm{C}$ for 1 minute and a final extension step at $72^{\circ} \mathrm{C}$ for 7 minutes. PCR purification was performed using a QIAquick PCR Purification Kit (Qiagen, Valencia, CA) according to the user manual. Samples were prepared for sequencing using $10 \mu \mathrm{L}$ of purified PCR amplicon (50 ng total) and $2 \mu \mathrm{L}$ of the sequencing primer $[1.7 \mu \mathrm{M}]$ and Sanger sequenced by ACGT Inc. (Germantown, MD). For $A D R B 1$, reference materials that were previously whole genome sequenced in the 1000 Genomes Project were obtained and genotyped. Variant information from the project was retrieved from https://www.ncbi.nlm.nih.gov/variation/ tools/1000genomes/ to confirm the accuracy of the $A D R B 1$ analytical validation study. 
The sequencing results were compared to the array genotyping results and were completely concordant for $100 \%$ accuracy. Based on the orthogonal method (e.g. DNA sequencing), the variant and reference allele used to calculate the assay's analytical sensitivity and specificity (Table 3 and Supplemental Table 2, Supplemental Digital Content 2, http:// links.lww.com/FPC/B332). The analytical sensitivity was $100 \%$ for the detection of variant alleles, with no reported false negatives. The analytical specificity was $100 \%$ for detection of reference alleles, with no false positive results reported (note at the 95th percentile, the confidence intervals varied based on variant frequencies). DNA samples were also used to assess intra- and inter-assay variation. In all, 14 samples obtained from Icahn School of Medicine at Mount Sinai were included in intra-assay validation. There were 18 samples included in the inter-assay validation which were comprised of the 14 Icahn School of Medicine at Mount Sinai samples plus four additional Coriell cell line samples that included the less frequent genotypes (NA07357, NA10847, NA18524, NA18563). In order to find these less frequent genotypes needed for validation, 189 total Coriell samples were genotyped successfully. These results are included in Supplemental Table 2, Supplemental Digital Content 2, http://links.lww.com/FPC/B332, to serve as reference materials for laboratories that may validate the same variants. The intra- (within) assay variation studies showed that all three replicates of the 14 samples ran on the same plate, were concordant with expected results. The inter- (between) assay variation studies showed that the 18 samples consistently yielded the same result across three separate runs. The stability had been previously established for the following conditions: DNA isolated from whole blood at room temperature after 1 week, refrigerated for 2 weeks, and frozen for 4 weeks, saliva after 5 years at room temperature, frozen indefinitely, and extracted DNA refrigerated and frozen for 5 and 15 years, respectively.

\section{Implementation}

The implementation of genetic testing to aid in personalized therapy has become more widespread. Applications for the variants detected with this assay may extend beyond the clinical care of individuals with hypertension and CKD as illustrated by the implementation efforts of networks like the Implementing Genomics in Practice (IGNITE) and APOL1 Long-Term Kidney Transplantation Outcomes (APOLLO) consortia. Further pragmatic trials are needed to determine clinical validity and utility. Understanding the underlying genetic architecture which predisposes an individual to develop chronic kidney disease allows us to build upon existing functional CKD classifications, which are presently based on estimate glomerular filtration rates and proteinuria. The implementation of genetic testing into clinical practice has the potential to augment drug selection, dose adjustment, improve the classification of CKD in patients, and more accurately predict the risk of progression. This testing serves as a complement to, not a replacement for, effective clinical practice.

\section{Supplementary Material}

Refer to Web version on PubMed Central for supplementary material. 


\section{Acknowledgements}

This study was supported by funding from NIH/NIDDK K08-DK107864 (Eadon), Indiana University's Grand Challenge Precision Health Initiative (Collins and Moorthi), and NIH/NHGRI U01-HG007762 (Pratt and Skaar).

\section{References:}

1. Gaedigk A, et al., The Pharmacogene Variation (PharmVar) Consortium: Incorporation of the Human Cytochrome P450 (CYP) Allele Nomenclature Database. Clin Pharmacol Ther, 2018 103(3): p. 399-401. [PubMed: 29134625]

2. Swen JJ, et al., Pharmacogenetics: from bench to byte--an update of guidelines. Clin Pharmacol Ther, 2011 89(5): p. 662-73. [PubMed: 21412232]

3. Spinasse LB, et al., Different phenotypes of the NAT2 gene influences hydralazine antihypertensive response in patients with resistant hypertension. Pharmacogenomics, 2014 15(2): p. 169-78. [PubMed: 24444407]

4. Pacanowski MA, et al., beta-adrenergic receptor gene polymorphisms and beta-blocker treatment outcomes in hypertension. Clin Pharmacol Ther, 2008 84(6): p. 715-21. [PubMed: 18615004]

5. Do AN, et al., The effects of genes implicated in cardiovascular disease on blood pressure response to treatment among treatment-naive hypertensive African Americans in the GenHAT study. J Hum Hypertens, 2016 30(9): p. 549-54. [PubMed: 26791477]

6. Gong Y, et al., Hypertension susceptibility loci and blood pressure response to antihypertensives: results from the pharmacogenomic evaluation of antihypertensive responses study. Circ Cardiovasc Genet, 2012 5(6): p. 686-91. [PubMed: 23087401]

7. Vandell AG, et al., G protein receptor kinase 4 polymorphisms: beta-blocker pharmacogenetics and treatment-related outcomes in hypertension. Hypertension, 2012 60(4): p. 957-64. [PubMed: 22949529]

8. Fava C, et al., 24-h ambulatory blood pressure is linked to chromosome 18q21-22 and genetic variation of NEDD4L associates with cross-sectional and longitudinal blood pressure in Swedes. Kidney Int, 2006 70(3): p. 562-9. [PubMed: 16788695]

9. Hiltunen TP, et al., Pharmacogenomics of hypertension: a genome-wide, placebo-controlled crossover study, using four classes of antihypertensive drugs. J Am Heart Assoc, 2015 4(1): p. e001521. [PubMed: 25622599]

10. Rimpela JM, et al., Replicated evidence for aminoacylase 3 and nephrin gene variations to predict antihypertensive drug responses. Pharmacogenomics, 2017 18(5): p. 445-458. [PubMed: 28353407]

11. Turner ST, et al., Genomic association analysis suggests chromosome 12 locus influencing antihypertensive response to thiazide diuretic. Hypertension, 2008 52(2): p. 359-65. [PubMed: 18591461]

12. Eadon MT, Kanuri SH, and Chapman AB, Pharmacogenomic studies of hypertension: paving the way for personalized antihypertensive treatment. Expert Rev Precis Med Drug Dev, 2018 3(1): p. 33-47. [PubMed: 29888336]

13. Eadon MT and Chapman AB, A Physiologic Approach to the Pharmacogenomics of Hypertension. Adv Chronic Kidney Dis, 2016 23(2): p. 91-105. [PubMed: 26979148]

14. Birdwell KA, et al., Clinical Pharmacogenetics Implementation Consortium (CPIC) Guidelines for CYP3A5 Genotype and Tacrolimus Dosing. Clin Pharmacol Ther, 2015 98(1): p. 19-24. [PubMed: 25801146]

15. Wilke RA, et al., The clinical pharmacogenomics implementation consortium: CPIC guideline for SLCO1B1 and simvastatin-induced myopathy. Clin Pharmacol Ther, 2012 92(1): p. 112-7. [PubMed: 22617227]

16. Dean L, Azathioprine Therapy and TPMT Genotype, in Medical Genetics Summaries, Pratt V, et al., Editors. 2012: Bethesda (MD).

17. Dean L, Clopidogrel Therapy and CYP2C19 Genotype, in Medical Genetics Summaries, Pratt V, et al., Editors. 2012: Bethesda (MD). 
18. Johnson JA, et al., Clinical Pharmacogenetics Implementation Consortium (CPIC) Guideline for Pharmacogenetics-Guided Warfarin Dosing: 2017 Update. Clin Pharmacol Ther, 2017.

19. Kottgen A, et al., New loci associated with kidney function and chronic kidney disease. Nature Genetics, 2010 42(5): p. 376-U34. [PubMed: 20383146]

20. Parsa A, et al., Genome-Wide Association of CKD Progression: The Chronic Renal Insufficiency Cohort Study. Journal of the American Society of Nephrology, 2017 28(3): p. 923-934. [PubMed: 27729571]

21. Parsa A, et al., APOL1 risk variants, race, and progression of chronic kidney disease. N Engl J Med, 2013 369(23): p. 2183-96. [PubMed: 24206458]

22. Leiherer A, et al., The value of uromodulin as a new serum marker to predict decline in renal function. J Hypertens, 2018 36(1): p. 110-118. [PubMed: 28858977]

23. Franceschini N, Frick A, and Kopp JB, Genetic Testing in Clinical Settings. Am J Kidney Dis, 2018 72(4): p. 569-581. [PubMed: 29655499]

24. Zhang J, et al., Analytical Validation of a Personalized Medicine APOL1 Genotyping Assay for Nondiabetic Chronic Kidney Disease Risk Assessment. J Mol Diagn, 2016 18(2): p. 260-6. [PubMed: 26773863]

25. Pratt VM, et al., Characterization of 107 genomic DNA reference materials for CYP2D6, CYP2C19, CYP2C9, VKORC1, and UGT1A1: a GeT-RM and Association for Molecular Pathology collaborative project. J Mol Diagn, 2010 12(6): p. 835-46. [PubMed: 20889555] 
Table 1:

Efficacy and Adverse Event Phenotype-Genotype Interactions

\begin{tabular}{|c|c|c|c|c|}
\hline Gene & Variant & $\begin{array}{l}\text { Relevant } \\
\text { Population }\end{array}$ & Genotyping Result & Predicted Phenotype \\
\hline \multirow[t]{3}{*}{$A D R B 1$} & \multirow[t]{3}{*}{ rs1801252, rs1801253 } & & 0 copies of $49 \mathrm{~S}-389 \mathrm{R}^{a}$ & Reduced Beta-Blocker Efficacy \\
\hline & & & 1 copy of 49S-389R & Standard Beta Blocker Efficacy \\
\hline & & & 2 copies of $49 \mathrm{~S}-389 \mathrm{R}$ & Increased Beta Blocker Efficacy \\
\hline \multirow[t]{3}{*}{ CYP2C19 } & \multirow{3}{*}{$\begin{array}{l}\text { rs4244285, rs4986893, } \\
\text { rs28399504, rs72552267, } \\
\text { rs41291556, rs6413438, } \\
\text { rs12248560 }\end{array}$} & & $* 1 /{ }^{*} 1,{ }^{*} 1{ }^{*}{ }^{*} 17$ & Standard Clopidogrel Efficacy \\
\hline & & & 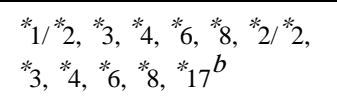 & Reduced Clopidogrel Efficacy \\
\hline & & & $* 17{ }^{*} 17$ & Increased Risk of Bleeding \\
\hline \multirow[t]{2}{*}{ CYP2C9 } & \multirow{2}{*}{$\begin{array}{l}\text { rs1799853, rs1057910, } \\
\text { rs28371686, rs9332131, } \\
\text { rs7900194, rs28371685 }\end{array}$} & & $* 1 /{ }^{*} 1$ & Standard Losartan Efficacy \\
\hline & & & ${ }^{*} 1 /{ }^{*} 2,{ }^{*},{ }^{*} 5,{ }^{*} 6,{ }^{*} 8,{ }^{*}{ }_{11} b$ & Reduced Losartan Efficacy \\
\hline \multirow[t]{3}{*}{ CYP2D6 } & \multirow{3}{*}{$\begin{array}{l}\text { rs16947, rs } 1135840, \text { rs } 35742686, \\
\text { rs3892097, rs1065852, rs5030655, } \\
\text { rs5030867, rs5030865(A), } \\
\text { rs5030656, rs1065852, rs1135840, } \\
\text { rs5030865(T), rs28371706, } \\
\text { rs61736512, rs59421388, } \\
\text { rs1135840, rs28371725 }\end{array}$} & & $\begin{array}{l}*_{1}^{*}{ }^{*} 1,{ }^{*} 2,{ }^{*} 9,{ }^{*} 10,{ }^{*} 17,{ }^{*} 2 / \\
*_{2}^{*},{ }^{*} 9,{ }^{*} 10,{ }^{*} 17,{ }^{*} 29,{ }^{*}{ }^{\prime} b\end{array}$ & Standard Metoprolol Efficacy \\
\hline & & & ${ }^{*} 1 /{ }^{*} 1 \mathrm{xN},{ }^{*} 1 /{ }^{*} 2 \mathrm{xN},{ }^{*} 2 /^{*} 2 \mathrm{xN} b$ & Reduced Metoprolol Efficacy \\
\hline & & & 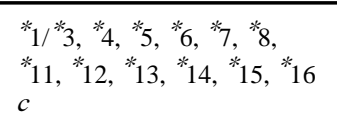 & $\begin{array}{l}\text { Increased Bradycardia with } \\
\text { Metoprolol }\end{array}$ \\
\hline \multirow[t]{2}{*}{ СУР3A4 } & \multirow[t]{2}{*}{ rs55785340, rs35599367 } & \multirow{2}{*}{$\begin{array}{l}\text { CYP3A5 } \\
* 3 /{ }^{*} 3,{ }^{*},{ }^{*} 7\end{array}$} & $*_{1}{ }^{*}{ }^{*} 1,{ }^{*} 1 /{ }^{*} 22$ & Standard Tacrolimus Dosing \\
\hline & & & $*^{*} 22{ }^{*} 22$ & Reduced Tacrolimus Dosing \\
\hline \multirow[t]{2}{*}{ CYP3A5 } & \multirow[t]{2}{*}{ rs776746, rs10264272, rs41303343 } & & $*_{3}{ }^{*} 3,{ }^{*} 6,{ }^{*} b$ & Standard Tacrolimus Dosing \\
\hline & & & $*_{1}^{*}{ }^{*} 1,{ }^{*} 3,{ }^{*} 6,{ }^{*} 7$ & Increased Tacrolimus Dosing \\
\hline \multirow[t]{3}{*}{$F 7$} & \multirow[t]{3}{*}{ rs6046 } & \multirow{3}{*}{$\begin{array}{l}\text { African } \\
\text { American }\end{array}$} & $\mathrm{G} / \mathrm{G}$ & Standard Amlodipine Efficacy \\
\hline & & & $\mathrm{G} / \mathrm{A}$ & Reduced Amlodipine Efficacy \\
\hline & & & $\mathrm{A} / \mathrm{A}$ & Poor Amlodipine Efficacy \\
\hline \multirow[t]{3}{*}{ FGF5/SH2B3/EBF1 } & \multirow[t]{3}{*}{ rs1458038, rs3184504, rs4551053 } & \multirow[t]{3}{*}{ Caucasian } & 0 efficacy alleles ${ }^{c}$ & Reduced Thiazide Efficacy \\
\hline & & & 1 or 2 efficacy alleles & Standard Thiazide Efficacy \\
\hline & & & 3 or more efficacy alleles & Increased Thiazide Efficacy \\
\hline \multirow[t]{3}{*}{ GRK4 } & \multirow[t]{3}{*}{ rs2960306, rs1024323 } & & 0 copies of $65 \mathrm{~L}-142 \mathrm{~V}^{a}$ & Increased Beta-Blocker Efficacy \\
\hline & & & 1 copy of $65 \mathrm{~L}-142 \mathrm{~V}$ & Standard Beta Blocker Efficacy \\
\hline & & & 2 copies of $65 \mathrm{~L}-142 \mathrm{~V}$ & Reduced Beta Blocker Efficacy \\
\hline \multirow[t]{2}{*}{ NAT2 } & \multirow{2}{*}{$\begin{array}{l}\text { rs1801279, rs1801280, rs1799930, } \\
\text { rs1799931 }\end{array}$} & & $*_{4} /{ }^{*} 4$ & Standard Hydralazine Efficacy \\
\hline & & & ${ }^{*} 4 /{ }^{*} 5,{ }^{*} 6,{ }^{*} 7,{ }^{*} 14 b$ & Increased Hydralazine Efficacy \\
\hline \multirow[t]{3}{*}{ NEDD4L } & \multirow[t]{3}{*}{ rs4149601 } & \multirow[t]{3}{*}{ Caucasian } & $\mathrm{G} / \mathrm{G}$ & Increased Diuretic Efficacy \\
\hline & & & G/A & Standard Diuretic Efficacy \\
\hline & & & $\mathrm{A} / \mathrm{A}$ & Reduced Diuretic Efficacy \\
\hline
\end{tabular}




\begin{tabular}{|c|c|c|c|c|}
\hline Gene & Variant & $\begin{array}{l}\text { Relevant } \\
\text { Population }\end{array}$ & Genotyping Result & Predicted Phenotype \\
\hline \multirow[t]{2}{*}{ NPHS1 } & \multirow[t]{2}{*}{ rs3814995 } & \multirow[t]{2}{*}{ Caucasian } & $\mathrm{G} / \mathrm{G}$ & Standard ARB Efficacy \\
\hline & & & $\mathrm{G} / \mathrm{A}$ or $\mathrm{A} / \mathrm{A}$ & Increased ARB Efficacy \\
\hline \multirow[t]{3}{*}{ SLCO1B1 } & \multirow[t]{3}{*}{ rs4149056, rs4149015 } & & ${ }^{*} 1 /{ }^{*} 1,{ }^{*} 1 /{ }^{*} 21,{ }^{*} 21 /{ }^{*} 21$ & Standard Simvastatin Dosing \\
\hline & & & $*_{1}^{*}{ }^{*} 5,{ }^{*} 1 /{ }^{*} 17,{ }^{*}{ }^{*}{ }^{*} 5$ & Reduced Simvastatin Dosing \\
\hline & & & ${ }^{*} 5 /{ }^{*} 17,{ }^{*} 17 /^{*} 17,{ }^{*} 17 I^{*} 21$ & Reduced Simvastatin Dosing \\
\hline \multirow[t]{3}{*}{$T P M T$} & \multirow{3}{*}{$\begin{array}{l}\text { rs } 1800462, \text { rs } 1800460 \text { and } \\
\text { rs } 1142345, \text { rs } 1800460, \text { rs } 1142345, \\
\text { rs } 1800584\end{array}$} & & $* 1 /{ }^{*} 1$ & Standard Azathioprine Dosing \\
\hline & & & \multirow{2}{*}{$\begin{array}{l}{ }_{1}^{*} 1 /^{*} 2,{ }^{*} 1{ }^{*} 3,{ }^{*} 2 /^{*} 2,{ }^{*} 2{ }^{*} 3, \\
{ }^{*} 3 /^{*} 3\end{array}$} & Reduced Azathioprine Dosing \\
\hline & & & & Reduced Azathioprine Dosing \\
\hline \multirow[t]{2}{*}{$V A S P$} & \multirow[t]{2}{*}{ rs 10995} & \multirow[t]{2}{*}{ Caucasian } & $\mathrm{A} / \mathrm{A}$ & Standard Thiazide Efficacy \\
\hline & & & $\mathrm{A} / \mathrm{G}$ or $\mathrm{G} / \mathrm{G}$ & Increased Thiazide Efficacy \\
\hline \multirow[t]{2}{*}{ VKORC1 } & \multirow[t]{2}{*}{ rs9923231 } & & $\mathrm{G} / \mathrm{G}$ & Standard Warfarin Sensitivity \\
\hline & & & $\mathrm{G} / \mathrm{A}$ or $\mathrm{A} / \mathrm{A}$ & Increased Warfarin Sensitivity \\
\hline \multirow[t]{2}{*}{ YEATS4 } & \multirow[t]{2}{*}{ rs 7297610} & \multirow{2}{*}{$\begin{array}{l}\text { African } \\
\text { American }\end{array}$} & $\mathrm{T} / \mathrm{T}$ or $\mathrm{T} / \mathrm{C} \mathrm{C} / \mathrm{C}$ & Standard Thiazide Efficacy \\
\hline & & & $\mathrm{C} / \mathrm{C}$ & Increased Thiazide Efficacy \\
\hline
\end{tabular}

Relevant population provided if results have only been identified in a specific population.

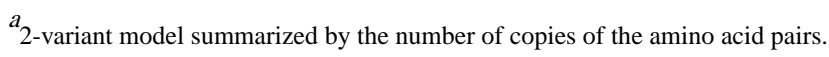

${ }^{b}$ An abbreviated list is provided due to the large number of possible genotyping results.

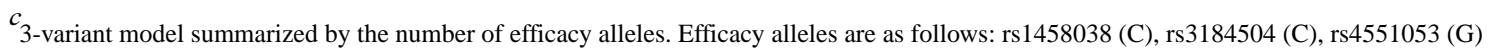


Table 2:

Genotype-Predicted Risks for Chronic Kidney Disease Progression

\begin{tabular}{|c|c|c|c|c|}
\hline Gene & Variant & $\begin{array}{l}\text { Relevant } \\
\text { Population* }\end{array}$ & Genotyping Result & $\begin{array}{l}\text { Predicted CKD } \\
\text { Progression Risk }\end{array}$ \\
\hline \multirow[t]{2}{*}{ APOLI } & \multirow[t]{2}{*}{ rs73885319, rs60910145, rs71785313 } & \multirow[t]{2}{*}{ African American } & 0 or 1 risk allele ${ }^{a}$ & Standard Risk \\
\hline & & & 2 or more risk alleles & Increased Risk \\
\hline \multirow[t]{2}{*}{ LINC00923 } & \multirow[t]{2}{*}{ rs653747 } & \multirow{2}{*}{$\begin{array}{l}\text { African American } \\
\text { non-diabetic }\end{array}$} & $\mathrm{C} / \mathrm{C}$ & Standard Risk \\
\hline & & & $\mathrm{C} / \mathrm{T}$ or $\mathrm{T} / \mathrm{T}$ & Increased Risk \\
\hline \multirow[t]{2}{*}{ SHROOM3 } & \multirow[t]{2}{*}{ rs17319721 } & \multirow[t]{2}{*}{ Caucasian } & $\mathrm{G} / \mathrm{G}$ & Standard Risk \\
\hline & & & $\mathrm{G} / \mathrm{A}$ or $\mathrm{A} / \mathrm{A}$ & Increased Risk \\
\hline \multirow[t]{2}{*}{ SHROOM3 } & \multirow[t]{2}{*}{ rs 4371638} & \multirow[t]{2}{*}{ African American } & $\mathrm{C} / \mathrm{C}$ & Standard Risk \\
\hline & & & $\mathrm{C} / \mathrm{T}$ or $\mathrm{T} / \mathrm{T}$ & Increased Risk \\
\hline \multirow[t]{2}{*}{ SHROOM3 } & \multirow[t]{2}{*}{ rs13146355 } & \multirow[t]{2}{*}{ Asian } & $\mathrm{G} / \mathrm{G}$ & Standard Risk \\
\hline & & & $\mathrm{G} / \mathrm{A}$ or $\mathrm{A} / \mathrm{A}$ & Increased Risk \\
\hline \multirow[t]{2}{*}{$S L C 3 A 2$} & \multirow[t]{2}{*}{ rs489381 } & \multirow[t]{2}{*}{ Caucasian } & $\mathrm{C} / \mathrm{C}$ & Standard Risk \\
\hline & & & $\mathrm{C} / \mathrm{A}$ or $\mathrm{A} / \mathrm{A}$ & Increased Risk \\
\hline \multirow[t]{2}{*}{ SLC3A2/LOC105369332 } & \multirow[t]{2}{*}{ rs2282538 } & \multirow[t]{2}{*}{ African American } & $\mathrm{G} / \mathrm{G}$ & Standard Risk \\
\hline & & & $\mathrm{G} / \mathrm{A}$ or $\mathrm{A} / \mathrm{A}$ & Increased Risk \\
\hline \multirow[t]{2}{*}{ UMOD } & \multirow[t]{2}{*}{ rs4293393 } & \multirow[t]{2}{*}{ African American } & $\mathrm{T} / \mathrm{T}$ or $\mathrm{T} / \mathrm{C}$ & Standard Risk \\
\hline & & & $\mathrm{C} / \mathrm{C}$ & Reduced Risk \\
\hline \multirow[t]{2}{*}{ UMOD } & \multirow[t]{2}{*}{ rs12917707 } & \multirow[t]{2}{*}{ Caucasian } & $\mathrm{C} / \mathrm{C}$ or $\mathrm{C} / \mathrm{A}$ & Standard Risk \\
\hline & & & $\mathrm{A} / \mathrm{A}$ & Reduced Risk \\
\hline \multirow[t]{2}{*}{ UMOD/PDILT } & \multirow[t]{2}{*}{ rs11864909 } & \multirow[t]{2}{*}{ Asian } & $\mathrm{G} / \mathrm{G}$ or $\mathrm{G} / \mathrm{A}$ & Standard Risk \\
\hline & & & $\mathrm{A} / \mathrm{A}$ & Reduced Risk \\
\hline
\end{tabular}

Relevant population provided if results have only been identified in a specific population.

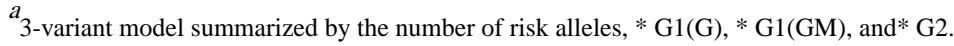


Table 3:

Analytical Sensitivity, Specificity, and Accuracy of OpenArray

\begin{tabular}{|c|c|c|c|c|}
\hline Gene & Variant & $\begin{array}{l}\text { Analytical } \\
\text { Sensitivity }\end{array}$ & $\begin{array}{l}\text { Analytical } \\
\text { Specificity }\end{array}$ & Accuracy \\
\hline \multirow{2}{*}{ ADRB1 } & c. $1165 \mathrm{G}>\mathrm{C}(\mathrm{rs} 1801253)$ & \multirow{2}{*}{$100 \%$ (95\% CI; 93-100) } & \multirow{2}{*}{$100 \%$ (95\% CI; 98-100) } & \multirow{2}{*}{$100 \%$} \\
\hline & c. $145 \mathrm{~A}>\mathrm{G}(\mathrm{rs} 1801252)$ & & & \\
\hline \multirow{3}{*}{ APOL1 } & c. $1072 A>G(r s 73885319)$ & \multirow{3}{*}{$100 \%$ (95\% CI; 91-100) } & \multirow{3}{*}{$100 \%$ (95\% CI; 98-100) } & \multirow{3}{*}{$100 \%$} \\
\hline & c.1200T>G (rs60910145) & & & \\
\hline & c.1212_1217delTTATAA (rs71785313) & & & \\
\hline EBF1 & g. $158411534 \mathrm{G}>\mathrm{A}(\mathrm{rs} 4551053)$ & $100 \%(95 \% \mathrm{CI} ; 57-100)$ & $100 \%$ (95\% CI; 74-100) & $100 \%$ \\
\hline FGF5 & g. $81164723 \mathrm{C}>\mathrm{T}(\mathrm{rs} 1458038)$ & $100 \%(95 \%$ CI; 51-100) & $100 \%$ (95\% CI; 61-100) & $100 \%$ \\
\hline \multirow{2}{*}{ GRK4 } & c.194G >T (rs2960306) & \multirow{2}{*}{$100 \%$ (95\% CI: 82-100) } & \multirow{2}{*}{$100 \%$ (95\% CI; 85-100) } & \multirow{2}{*}{$100 \%$} \\
\hline & c. $329 \mathrm{C}>\mathrm{T}(\mathrm{rs} 1024323)$ & & & \\
\hline LINC00923 & g. $97659878 \mathrm{C}>\mathrm{T}(\mathrm{rs} 653747)$ & $100 \%$ (95\% CI; 79-100) & $100 \%$ (95\% CI; 72-100) & $100 \%$ \\
\hline \multirow{4}{*}{ NAT2 } & c. $857 \mathrm{G}>\mathrm{A}(\mathrm{rs} 1799931)$ & \multirow{4}{*}{$100 \%$ (95\% CI; 97-100) } & \multirow{4}{*}{$100 \%$ (95\% CI; 95-100) } & \multirow{4}{*}{$100 \%$} \\
\hline & c.191G>A (rs1801279) & & & \\
\hline & c.590G>A (rs1799930) & & & \\
\hline & c. $341 \mathrm{~T}>\mathrm{C}(\mathrm{rs} 1801280)$ & & & \\
\hline NEDD4L & c. $24 \mathrm{G}>\mathrm{A}(\mathrm{rs} 4149601)$ & $100 \%(95 \% \mathrm{CI} ; 57-100)$ & $100 \%(95 \% \mathrm{CI} ; 83-100)$ & $100 \%$ \\
\hline NPHS1 & g. $35851310 \mathrm{C}>\mathrm{T}(\mathrm{rs} 3814995)$ & $100 \%(95 \% \mathrm{CI} ; 65-100)$ & $100 \%(95 \%$ CI; 65-100) & $100 \%$ \\
\hline \multirow{3}{*}{ SHROOM3 } & c. $168+11474 \mathrm{G}>\mathrm{A}(\mathrm{rs} 17319721)$ & \multirow{3}{*}{$100 \%$ (95\% CI; 57-100) } & \multirow{3}{*}{$100 \%(95 \% \mathrm{CI} ; 65-100)$} & \multirow{3}{*}{$100 \%$} \\
\hline & c.169-36495T>C (rs4371638) & & & \\
\hline & c. $168+54767 \mathrm{G}>\mathrm{A}(\mathrm{rs} 13146355)$ & & & \\
\hline SH2B3 & c.784T $>C($ rs 3184504$)$ & $100 \%(95 \%$ CI; 61-100) & $100 \%$ (95\% CI; 72-100) & $100 \%$ \\
\hline SLC3A2 & c. $1066-41 \mathrm{~A}>\mathrm{G}(\mathrm{rs} 489381)$ & $100 \%(95 \%$ CI; 77-100) & $100 \%(95 \% \mathrm{CI} ; 72-100)$ & $100 \%$ \\
\hline SLC3A2/LOC105369332 & g. $62832260 \mathrm{C}>\mathrm{T}(\mathrm{rs} 2282538)$ & $100 \%(95 \%$ CI; 34-100) & $100 \%(95 \% \mathrm{CI} ; 84-100)$ & $100 \%$ \\
\hline \multirow{2}{*}{ UMOD } & $\mathrm{c}-680 \mathrm{~T}>\mathrm{C}(\mathrm{rs} 4293393)$ & \multirow{2}{*}{$100 \%$ (95\% CI; 68-100) } & \multirow{2}{*}{$100 \%$ (95\% CI; 90-100) } & \multirow{2}{*}{$100 \%$} \\
\hline & c. $-214 \mathrm{C}>\mathrm{A}(\mathrm{rs} 12917707)$ & & & \\
\hline UMOD/PDILT & c.203-4666G>A (rs11864909) & $100 \%$ (95\% CI; 34-100) & $100 \%(95 \% \mathrm{CI} ; 85-100)$ & $100 \%$ \\
\hline VASP & c.*719G $>$ A (rs10995) & $100 \%(95 \%$ CI; 70-100) & $100 \%(95 \%$ CI; 57-100) & $100 \%$ \\
\hline YEATS4 & g. $69430244 \mathrm{C}>\mathrm{T}(\mathrm{rs} 7297610)$ & $100 \%(95 \%$ CI; 34-100) & $100 \%(95 \% \mathrm{CI} ; 85-100)$ & $100 \%$ \\
\hline
\end{tabular}

